
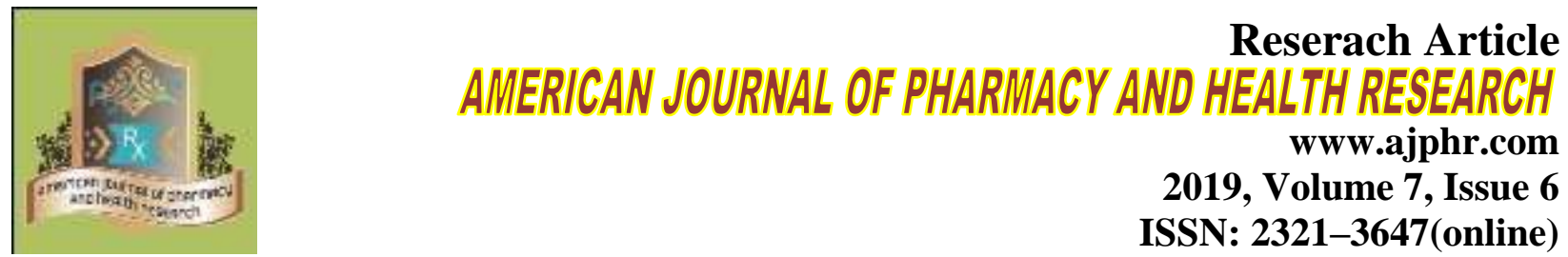

2019, Volume 7, Issue 6

ISSN: 2321-3647(online)

\title{
Development, Characterization \& Statistical Optimization of Matrix Type Transdermal Patches of Almotriptan Malate for The Treatment of Migraine
}

\author{
Akila RM ${ }^{1 *}$, Naseem AK ${ }^{1}$ \\ College of Pharmacy, Department of Pharmaceutics, Sri Ramakrishna Institute of Paramedical \\ Sciences, Coimbatore
}

\begin{abstract}
The present work is focused on the development, evaluation and statistical optimization of almotriptan malate transdermal patches. Almotriptan malate is used as anti-migraine drug and reported to have the best-sustained pain free rate and the lowest adverse events rate of all the triptans and thus making interest in the development of new route for the therapeutic administration of the drug, So, the present research work was aimed to develop matrix type transdermal patches using polymers HPMC K15M, ERS100 and EC and plasticizers DBP and PEG400 by solvent casting method. This formulation would help to avoid non-compliance of the tablet dosage form The IR spectra of the drug and physical mixtures of drug-polymer confirmed the absence of incompatibility between drug and polymers. The in-vitro drug release studies revealed that patches made of HPMC K15M-ERS 100 with DBP as plasticizer was considered as the best formulation and incorporated in $2^{2}$ factorial design and evaluated for further studies. The concentration of HPMC K 15M and ERS 100 are chosen as the independent variables. Drug content, \%moisture absorption \%, moisture loss, and cumulative \% drug released at $12 \mathrm{~h}$ are chosen as dependent variables. The drug release from transdermal patches followed zero order kinetics. Various physicochemical parameters of the patches were investigated. The current research work of transdermal patches of almotriptan malate may provide sustained delivery for prolonged periods in the management of migraine, which can be a good way to bypass the extensive hepatic first-pass metabolism.
\end{abstract}

Keywords: migraine, almotriptan malate, transdermal patches, solvent casting, $2^{2}$ factorial design

*Corresponding Author Email: ktakila@yahoo.co.in

Received 10 June 2019, Accepted 17 June 2019

Please cite this article as: Akila RM et al., Development, Characterization \& Statistical Optimization of Matrix Type Transdermal Patches of Almotriptan Malate for The Treatment of Migraine. American Journal of Pharmacy \& Health Research 2019. 


\section{INTRODUCTION}

A Migraine is a primary headache disorder consisting of moderate to severe intensity headache associated with some combination of severe disability and neuro-autonomic dysfunction such as visual or other types of aura, gastric stasis, poor absorption from the small bowel, nausea, vomiting, vertigo that worsens with movement, sonophobia, and photophobia. These clinical features lead to challenges in treatment, especially given the wide availability of various categories of oral medications, which are not ideally suited for those migraineurs with nausea, vomiting, and poor gastrointestinal absorption. The majority of migraineurs experience nausea and many experience vomiting with their attacks. For many, these clinical features have a negative impact on how, if, and when they treat their migraine[1]. This suggests that a non-oral route of delivery may prove optimal in these patients. Additionally, new studies suggest that migraineurs experience an underlying gastric stasis during and between migraine attacks, which may slow gastric transit time and compromise subsequent small bowel absorption of the medication [2]. Therefore, drug delivery systems that rely on gastric motility and intestinal absorption, such as oral medication, may prove slow, ineffective or inconsistently effective in some patients. Injection and nasal delivery systems for migraineurs do exist and they bypass gastrointestinal absorption [3] however, these approaches may be associated with limiting adverse events for some patients. Injectable sumatriptan may be associated with injection-site irritation and pain as well as increased adverse events (i.e. chest and neck discomfort, warm feelings, dizziness, drowsiness, increased nausea, and paraesthesias) compared with other delivery systems. These adverse events may be so severe that patients hesitate to use the medication or may delay taking it, or use it as rescue therapy once other treatments have failed. Some nasal spray delivery systems for migraine are easy to use and can work more quickly than tablets, but can be associated with bad taste thereby exacerbating nausea and vomiting in some patients. Additionally, nasally delivered triptans rely to some extent on gastric absorption due to swallowing a significant amount of the drug that drains down the posterior nasal cavity. This may prove problematic for those migraineurs who experience nausea or gastric stasis with their attacks. Importantly, some patients do not seem to like nasal sprays for the treatment of a migraine. Recent developments in drug delivery systems show that a new migraine patch may address many of these concerns for patients. The patches offer the patient many clinical benefits including a convenient, non-invasive delivery of medication that bypasses the gastrointestinal tract and hepatic first-pass metabolism. This delivery may prove to be the route of choice for 
patients experiencing symptoms consistent with gastrointestinal dysfunction (nausea, vomiting, gastric stasis) associated with a migraine. Rapid, consistent and precise delivery of stable and optimal therapeutic plasma concentrations of almotriptan malate via the transdermal patch, without exposing patients to exceedingly high or variable concentrations, may lead to superior efficacy and a lower incidence of triptan-related adverse events[4].

\section{MATERIALS AND METHOD}

\section{Materials}

Almotriptan malate was procured from Azakem laboratories, Hyderabad as a gift sample. Hydroxypropyl methylcellulose K-15M (HPMC) and Eudragit RS 100(ERS) were purchased from Yarrow Chem. Products, Mumbai. Ethyl cellulose, Polyethylene Glycol 400 (PEG) were obtained from Hi-Media Laboratories, Private Ltd, Mumbai. Dibutyl phthalate and methanol were purchased from SDFCL, Mumbai. All other materials and chemicals used were of either pharmaceutical grade or analytical reagent grade (AR grade).

\section{Methods}

\section{Preformulation Studies}

Before developing the drug into the transdermal patch, preformulation studies were carried out to establish the physicochemical characteristics of the drug, almotriptan malate.

\section{Solubility of Almotriptan Malate}

The solubility of Almotriptan Malate was tested in various solvents, $10 \mathrm{mg}$ of the drug was dissolved in $10 \mathrm{ml}$ of each solvent at room temperature. The solubility was observed by visual inspection.

\section{Development of Calibration Curve of Almotriptan Malate[5]}

Stock solution $(100 \mu \mathrm{g} / \mathrm{ml})$ was prepared in phosphate buffer $\mathrm{pH} 7.2$ Using this solution, serial dilutions were done and solutions of concentration of 2,4,6,8 and $10 \mu \mathrm{g} / \mathrm{ml}$ were prepared and absorbance was measured against blank of $\mathrm{pH} 7.2$ phosphate buffer in u.v. spectrophotometer at $227 \mathrm{~nm}$.

\section{Compatibility between drug and excipients [6]}

Compatibility study of drug with the excipients was determined by Fourier to transform infrared (FTIR) spectroscopy(Jasco FTIR 4100).

\section{Formulation of transdermal patches of Almotriptan Malate [7]}

In the present research work, matrix-type transdermal patches of Almotriptan malate(A1, B1, C1-6.25mg and A2, B2, C2-12.5mg) were prepared by solvent evaporation technique using 
different polymers such as HPMC K15M, ERS100, EC in presence of plasticizer DBP or PEG400. Solvents either DCM and methanol or water is used. Polymeric solutions were prepared by dissolving the polymers in the solvent and specified amount of drug and plasticizer were added and stirred well in a magnetic stirrer for 30min to get a homogenous solution. Then the solution was poured into a Petri plate which was lubricated with glycerine, entrapped air bubbles were removed and dried at room temperature for $24 \mathrm{~h}$ for solvent evaporation. The rate of solvent evaporation was controlled by inverting a glass funnel over the Petri plate. After $24 \mathrm{~h}$ the patches were removed by peeling and cut into the circle of radius $2.1 \mathrm{~cm}$ having surface area $13.85 \mathrm{~cm}^{2}$. These patches were kept in a desiccator for 2 days for further drying and wrapped in aluminum foil, packed in self-sealing covers.

\section{Characterization of transdermal patches of Almotriptan Malate}

\section{Physical Appearance}

All the prepared formulations were visually inspected for colour, clarity, flexibility and smoothness

\section{Thickness [8]}

The thickness of the drug loaded patches were measured at 3 different points by using a digital micrometer and the mean value was calculated.

\section{Folding endurance [9]}

A strip of the specific area was cut evenly and repeatedly folded at the same place till it broke. The number of times the film can be folded-up at the same place while not breaking gave the worth of folding endurance.

\section{Drug Content[10]}

A specified area of the film was cut and was dissolved in suitable solvent in specific volume. Then the solution was filtered through a filter medium and analyzed for the drug content using uv spectrophotometer at $227 \mathrm{~nm}$.

\section{Weight Uniformity [11]}

The prepared patches were dried at $60^{\circ} \mathrm{C}$ for $4 \mathrm{~h}$ before testing. 5 patches were randomly selected and subjected to weight uniformity tests by weighing individually in an electronic digital balance and the average weight was calculated.

\section{Surface pH [12]}

Surface $\mathrm{pH}$ of the patches was determined by using $\mathrm{pH}$ meter.3 patches of every formulation were allowed to swell by keeping in touch with double distilled water for $1 \mathrm{~h}$ in glass tubes. The 
surface $\mathrm{pH}$ was then noted by bringing a combined glass electrode near the surface of the patch and allowing it to equilibrate for 1 minute.

\section{Swelling Index [13]}

A specified area of the film was weighed and put in a Petri dish containing $10 \mathrm{ml}$ of double distilled water and was allowed to imbibe. Increase in weight of the patch was determined at preset time intervals until a constant weight was observed.

The degree of swelling $(\mathrm{S})$ was calculated using the formula:

$\mathrm{S}(\%)=\mathrm{Wt}-\mathrm{Wo} / \mathrm{Wo} \times 100$

Where $\mathrm{S}$ is percent swelling, Wt is the weight of patch at time $\mathrm{t}$ and Wo is the weight of patch at time 0 .

\section{\% Moisture content[14]}

The prepared films were weighed individually and to be kept in a desiccator containing fused calcium chloride at room temperature for $24 \mathrm{~h}$. After $24 \mathrm{~h}$ the films were taken out and reweighed. The moisture content was calculated using the formula:

$\%$ moisture content $=$ Initial weight - Final weight $/$ Final weight $\times 100$

\section{$\%$ Moisture loss [15]}

The weighed films were placed in a desiccator at room temperature for $24 \mathrm{~h}$ containing a saturated solution of potassium chloride in order to maintain $84 \%$ RH. After $24 \mathrm{~h}$ the films were taken out, reweighed and determined for \% moisture uptake using the following formula, $\%$ moisture uptake=Final weight - Initial weight $/$ Initial weight $\mathrm{x} 100$

\section{Water vapour transmission rate [8]}

About $1 \mathrm{~g}$ of fused calcium chloride was taken in glass vials of equal diameter and the polymeric patches measuring $1 \mathrm{~cm} 2$ area were fixed over the brim with the help of an adhesive. They were weighed accurately and initial weight was recorded, and then kept in a closed desiccator containing the saturated solution of potassium chloride to maintain $80-90 \% \mathrm{RH}$. The cells were taken out and weighed every $24 \mathrm{~h}$ for 1 week. The amount and rate of water vapour transmitted were calculated by the difference in weight using the formula:

Water vapour transmission rate $=$ Final weight- Initial weight/Time $\mathrm{x}$ area

\section{In-vitro drug release study [16]}

In-vitro drug release study was carried out through dialysis membrane-50(Hi-Media) by using a Franz diffusion cell with a cross-sectional area of $13.84 \mathrm{~cm}^{2}$. The formulated patches were cut into $2.1 \mathrm{~cm}$ radius and placed over the dialysis membrane in such a way that the drug releasing surface face towards the receptor compartment. The receptor compartment was filled with 
phosphate buffer $\mathrm{pH}$ 7.4. The whole assembly was fixed on a hot plate magnetic stirrer and the solution in the receptor compartment was constantly and continuously blended with magnetic beads at $50 \mathrm{rpm}$ and the temperature was maintained at $37 \pm 0.5^{\circ} \mathrm{C}$ by circulating the constant temperature water through outer jacket of diffusion cells.

$1 \mathrm{ml}$ of the sample was withdrawn at different time intervals over $12 \mathrm{hrs}$ and analyzed for drug content spectrophotometrically at $227 \mathrm{~nm}$. The receptor phase was replenished with an equal volume of phosphate buffer $\mathrm{pH} 7.4$ at each sample withdrawal.

\section{Full factorial design [17]}

Minitab 18 was used for the effect of analysis of each variable on the designated response. Surface plots were made for the analysis of each response coefficient for its statistical significance. The significant polynomial equations were used to validate the statistical design. From the above formulations prepared, the best one (A1 \& A2) was selected after their characterization, and were subjected for experimental design and data analysis. A $2^{2}$ full factorial design was selected for the above study in which two independent factors were evaluated, each at 2 levels. So, totally 4 runs were performed (Table 1). The different concentration of the polymers HPMC K 15M and ERS 100 were chosen as independent variables. Drug content, moisture absorption, moisture loss and cumulative \% drug release at $12 \mathrm{hr}$ were taken as dependent variables.

The effect of mixture component on dependent variables were modelled using the following equation

$Y=b_{0}+b_{1} X_{1}+b_{2} X_{2}+b_{12} X_{1} X_{2}+b_{11} X_{12}+b_{22} X_{22}$

Where $\mathrm{Y}$ is predicated response, $\mathrm{b}_{0}$ is the arithmetic mean of all responses, $\mathrm{b}_{1} \& \mathrm{~b}_{2}$ are the estimated coefficient for factors A \& B respectively.

Table 1: Two factor two level factorial design

\section{Stability studies [18]}

\begin{tabular}{lll}
\hline Independent factors & $\begin{array}{l}\text { Levels } \\
(-)\end{array}$ & $(+)$ \\
\hline HPMC K 15M (A) & $400 \mathrm{mg}$ & $600 \mathrm{mg}$ \\
ERS (B) & $400 \mathrm{mg}$ & $600 \mathrm{mg}$ \\
\hline
\end{tabular}

Stability studies were conducted according to ICH guidelines by storing the TDDS samples at $40+0.5^{\circ} \mathrm{C}$ and $75+5 \% \mathrm{RH}$ for 60 days. The samples were withdrawn at 0,30 and 60 days and analyze suitably for the drug content and for the \% cumulative drug release.

In-vitro drug release kinetic study [19] 
The kinetic study was done using a software Microsoft Office Excel Add-In. The in-vitro drug permeation of optimized formulation was fitted to zero order kinetics (cumulative amount of drug released versus time), Higuchi model (cumulative percentage drug release versus square root of time), Korsmeyer-Pappas model (log cumulative percentage drug release versus log time) to assess the kinetic modeling of drug release and the model with the higher correlation coefficient( i.e higher $\mathrm{R}^{2}$ ) was considered to be the best fit model.

\section{RESULTS AND DISCUSSION}

\section{Preformulation studies}

Solubility studies revealed that the almotriptan malate was soluble readily in various solvents such as water, dichloromethane, methanol, and phosphate buffer $\mathrm{pH}$ 6.5. Calibration curve of Almotriptan malate was constructed and found to be linear.

The IR spectra of Almotriptan malate and the physical mixture of drug with excipients showed that the drug and excipients are chemically compatible as there were no abnormal peaks. (Figure $1-4)$

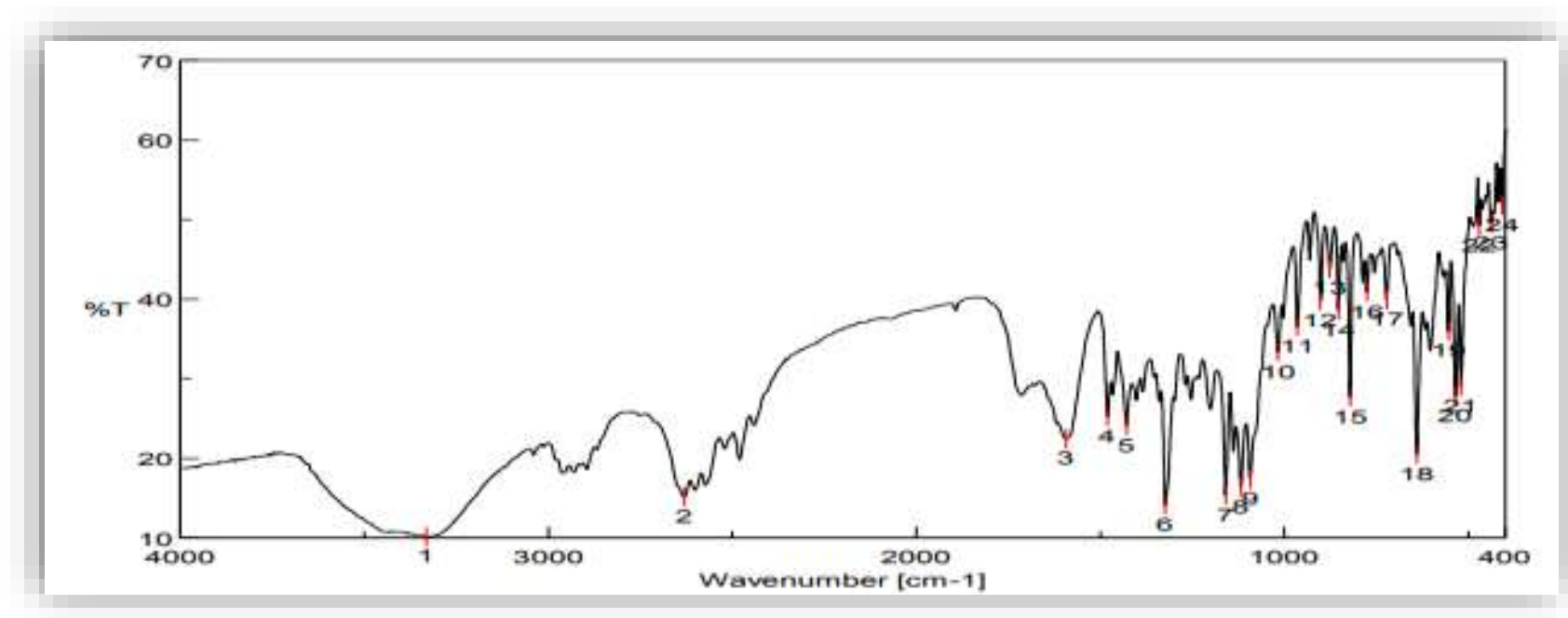

Figure 1: IR spectrum of drug Almotriptan malate 


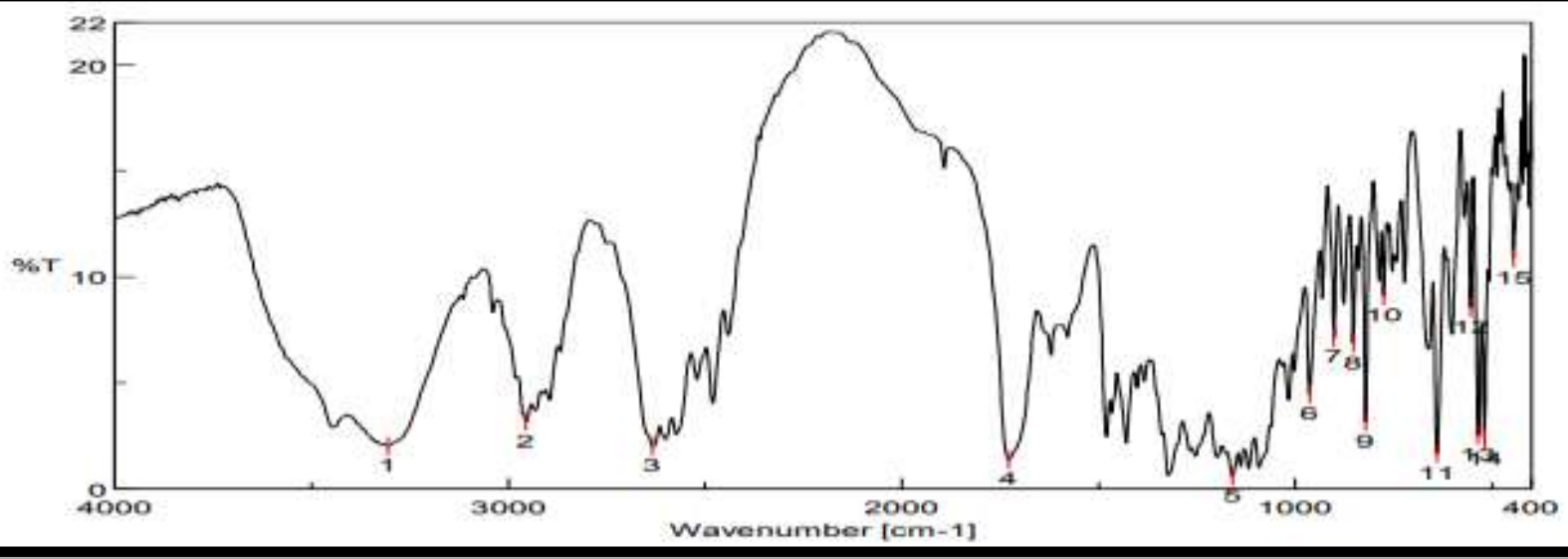

Figure 2: IR spectrum of drug Almotriptan malate with polymers HPMC K15M \& ERS100

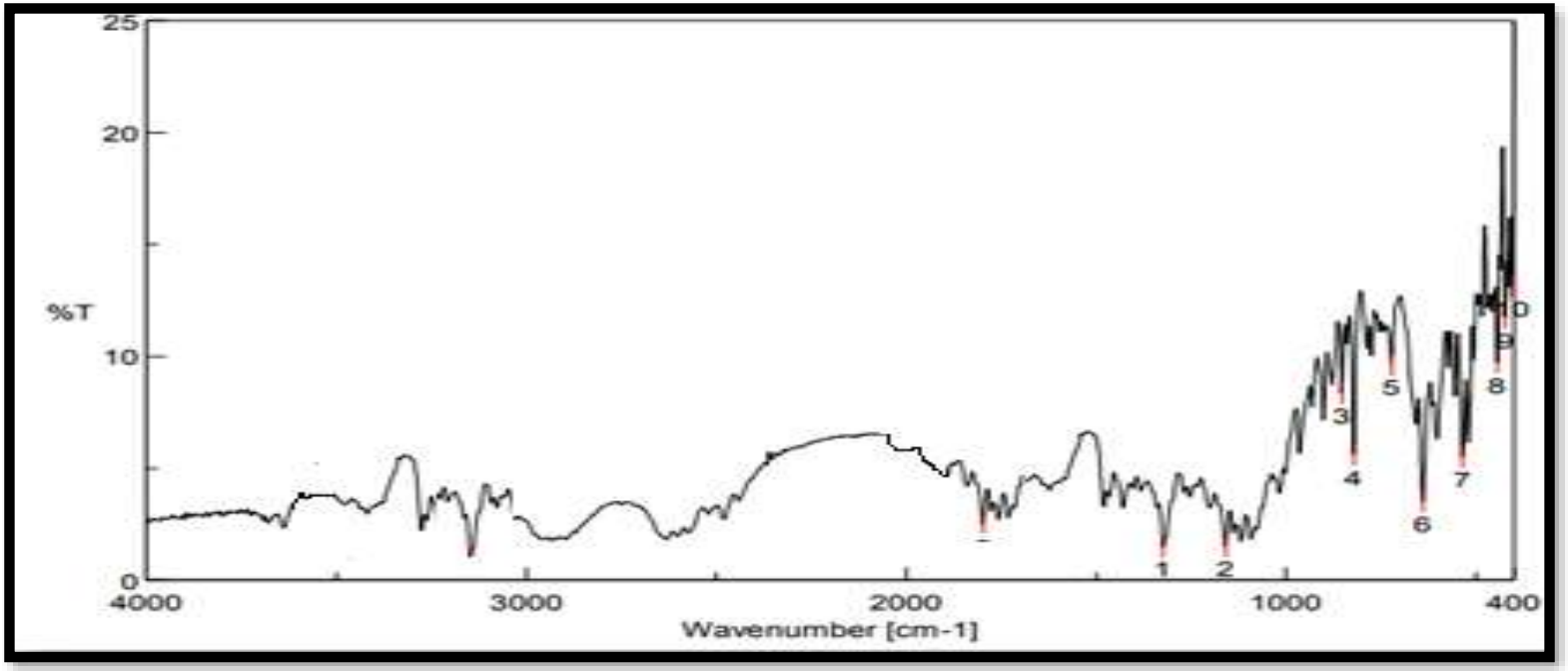

Figure 3: IR spectrum of drug Almotriptan malate with polymers HPMC K15M \& EC

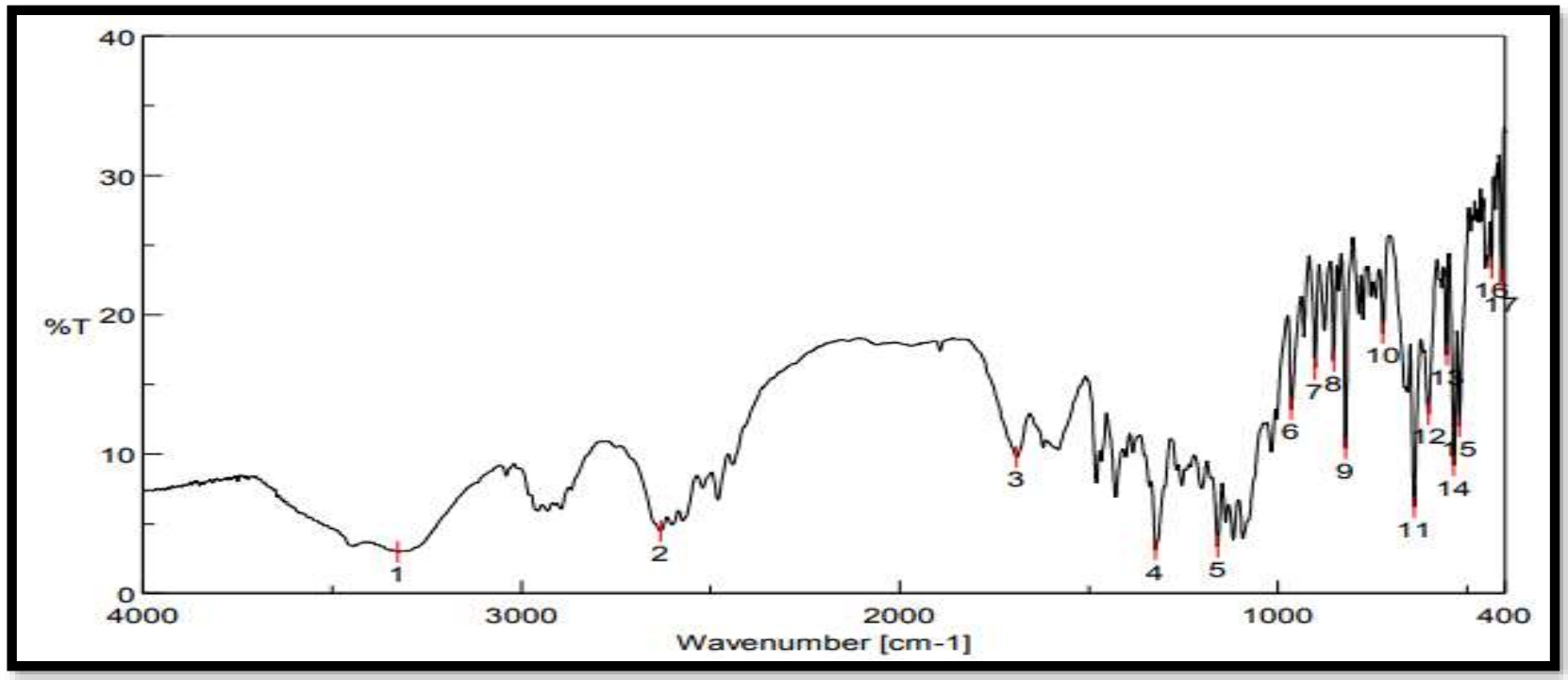

Figure 4: IR spectrum of drug Almotriptan malate with polymers HPMC K15M 


\section{Formulation of transdermal patches of Almotriptan Malate [20] \\ Calculation of total drug loading}

The formulation of the patch was made in such a way that each small circular patch of the $2.1 \mathrm{~cm}$ radius(which is the radius of the Franz diffusion cell) contains $6.25 \mathrm{mg}$ or $12.5 \mathrm{mg}$ of the drug. The total amount of drug to be loaded in the patch was calculated by measuring the total area of the Petri dish in which the patch will be cast. The calculation was done as follows:

The total amount of drug to be loaded $=($ area of the Petri dish in which the patch is molded $\mathrm{x}$ desired drug amount)/area of the small circular patch.

As per the methodology, the transdermal patches were prepared by solvent evaporation technique.

\section{Characterization of transdermal patches of Almotriptan Malate}

Physical appearances of the films were evaluated. All the films were easily removable from the mold without any recrystallization. All the systems were thin, flexible, smooth and without the entrapment of air but formulation having only the polymer HPMC K 15M was found to be clear and transparent. The method adopted for casting the systems was found to be satisfactory.

The thickness of patches ranged from 0.103 to $0.332 \mathrm{~mm}$ and gave reproducible results. Folding endurance test indicated that the patches did not show any cracks even after folding 300 times and thus showing good elasticity, maintaining the integrity with general skin folding. The folding endurance measures the potential of a patch to withstand rupture.

\section{Drug Content}

The drug content in each small circular patches was analyzed spectrophotometrically and it was observed that all the formulations showed a satisfactory drug content values ranging from 88 $99 \%$ ensures the uniform reproducible sustained release of the drug from the patch.

\section{Weight Uniformity}

From each batch, the weight of five patches was taken on a digital balance. It was observed that the weight of the entire film sample in each formulation was uniform.

\section{Surface pH}

The surface $\mathrm{pH}$ for all the formulations was well within the optimum range of 5-6 and hence no skin irritation was expected to occur after applications of the patches.

\section{$\%$ Swellability index}

The percentage of swelling of Almotriptan Malate loaded HPMC K 15M-ERS 100 patches was around 30 and the other formulations also showed considerable swelling. The hydrophilic 
polymer showed considerable swelling, as it increased the surface wettability and consequently water penetration within the matrix.

In the swelling study it was observed that as time increased, the swelling index was also increased because weight gain by the patch was increased proportionally with the rate of hydration up to certain time. The consequence of water uptake could be the formation of empty spaces within the patch that could make its structure less resistant to mechanical stresses.

\section{Percentage of Moisture Absorption}

Percentage moisture absorption for HPMC-ERS 100, HPMC-EC and HPMC alone was found in the range of $14.72 \%, 9.5 \%$, and $4.94 \%$ respectively; this may be due to hydrophilic and hydrophobic nature of the polymer.

The lower moisture content in the formulations made the patches to remain stable and become a completely dried and brittle film and also protects the material from microbial contamination and bulkiness.

\section{Percentage Moisture Loss}

Percentage moisture absorption for HPMC-ERS 100, HPMC-EC and HPMC alone was found in the range of $14.72 \%, 9.5 \%$, and $4.94 \%$ respectively; this may be due to hydrophilic and hydrophobic nature of the polymer.

The lower moisture content in the formulations made the patches to remain stable and become a completely dried and brittle film and also protects the material from microbial contamination and bulkiness.

\section{Percentage of Moisture Loss}

From moisture loss study it was found that formulation showed a maximum amount of moisture loss due to HPMC K $15 \mathrm{M}$ as it undergoes moisture loss in dry condition. In spite of the moisture loss, patches were found to maintain their physical stability.

The low percentages of moisture loss help them to remain stable and free from completely drying and brittle.

\section{Water Vapour Transmission Rate}

Patches having ERS 100 as the hydrophobic polymer showed least transmission rate than that of patches with EC. All the formulations were permeable to water vapour. Low water vapor transmission rate also indicates a high degree of stability even in high humid conditions.

\section{In-vitro drug release study}

The cumulative percent drug release was higher in case of Eudragit containing polymer matrix as it released $3.39 \mathrm{mg} / \mathrm{cm} 2 / 12 \mathrm{hrs}$ and $6.08 \mathrm{mg} / \mathrm{cm} 2 / 12 \mathrm{hrs}$ for low dose $(6.25 \mathrm{mg}) \&$ high dose 
$(12.5 \mathrm{mg}$ ) of Almotriptan Malate (Figure $5 \&$ 6). The reason for high release from ERS 100 polymer could be explained by the hydrophobic nature of this polymer and the existence of the quaternary ammonium groups which could affect the release from the patches because of the hydration and swelling of the patches. Eudragit (Polymethyl methacrylate) is known to have larger cavity size in its polymeric network and thus it may involve a faster mode of diffusion of Almotriptan Malate from the ERS 100: HPMC K 15M formulations as compared to the EC: HPMC K 15M formulations.

Release of the drug from transdermal patches is controlled by the chemical properties of the drug and delivery form, as well as physiological and physicochemical properties of the biological membrane. The process of drug release in most controlled release devices is governed by diffusion and the polymer matrix has strong influence on the diffusivity as the motion of a small molecule is restricted by the three-dimensional network of polymer chains

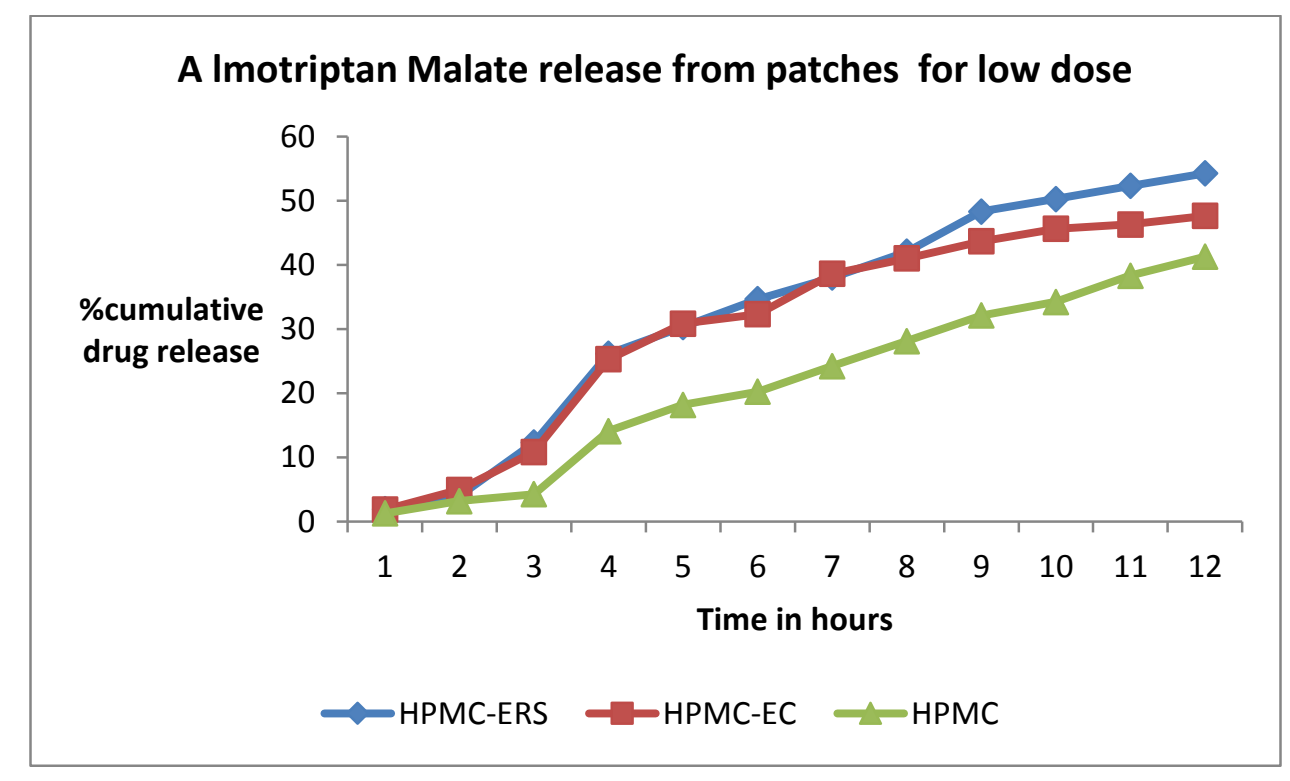

Figure 5: In-vitro drug release studies for lower dose $(6.25 \mathrm{mg})$ 


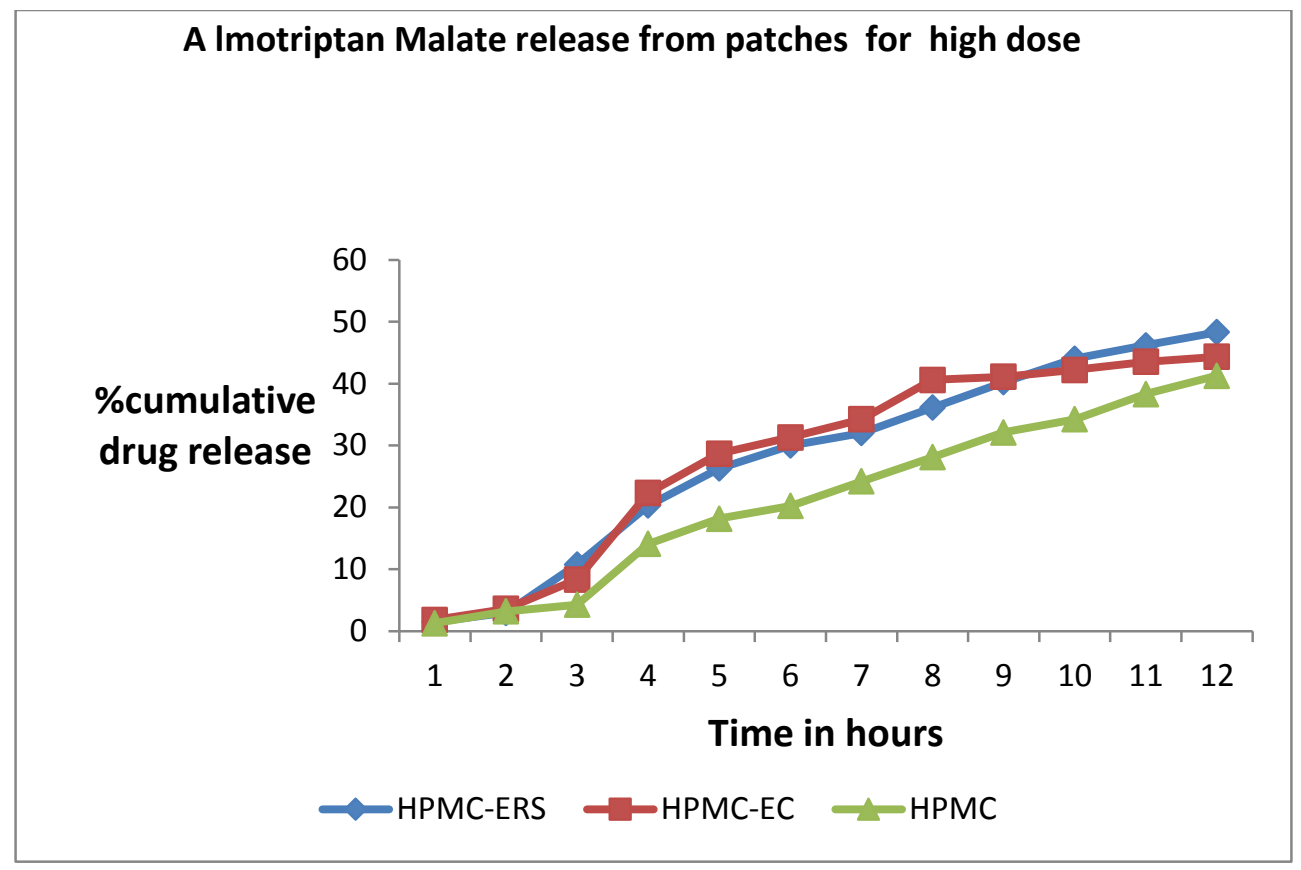

Figure 6: In-vitro drug release studies for higher dose(12.5mg)

\section{Full Factorial Design}

The aim of the present work was to achieve optimized formulations for Almotriptan loaded transdermal patches by determining the effects of some important factors (variables) and their interactions during the process of preparation. Among all combinations HPMC K 15M: ERS 100 loaded with Almotriptan Malate gave better results. Hence these formulations were selected and incorporated in $2^{2}$ factorial design and evaluated for further studies. Two of the most significant factors (concentration of HPMC K 15M and ERS 100) had been chosen as the independent variables. In the next step, for determining the low and high levels of each factor, some formulations were made. According to a $2^{2}$ factorial design and considering these two variables, 4 experiments each for low and high doses of Almotriptan had been performed. The effect of these variables on drug content, moisture absorption, moisture loss and the cumulative amount of drug release at $12 \mathrm{hr}$ were investigated as optimization response parameters in the current study.The relationship between the dependent and various independent variables was further elucidated using 3-D response plot (Figure 7-14)

It provides flexibility and making each response significant individually. Optimization aided in understanding the interaction of formulation parameters, which can be exemplified by the increase in both the polymer concentration decreases drug release and it was found that concentration of HPMC K 15M was found to have an individual effect on moisture uptake and loss i.e. when its concentration increased, moisture absorption and loss also increased. 
Surface Plot of Drug content vs ERS 100, HPMC K 15M

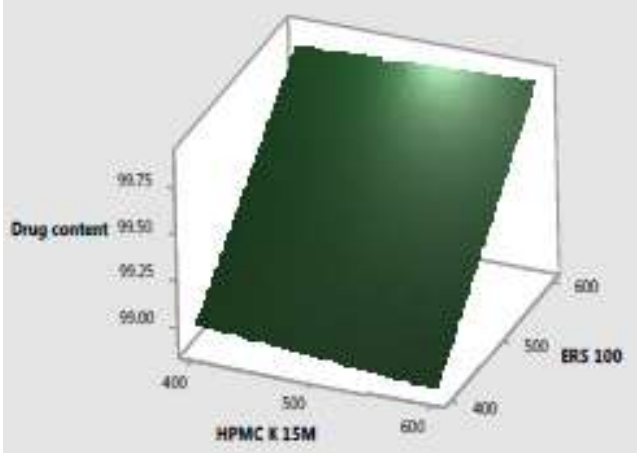

Figure 7:

Surface Plot of Moisture loss vs ERS 100, HPMC K 15M

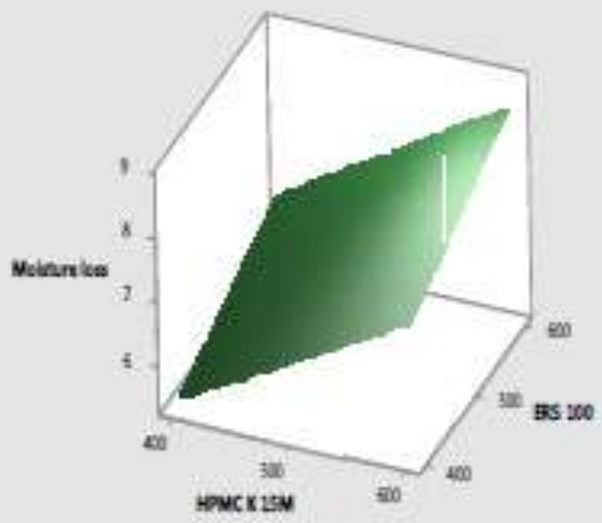

Figure 9:
Surface Plot of Moisture absorption vs ERS 100, HPMC K 15M

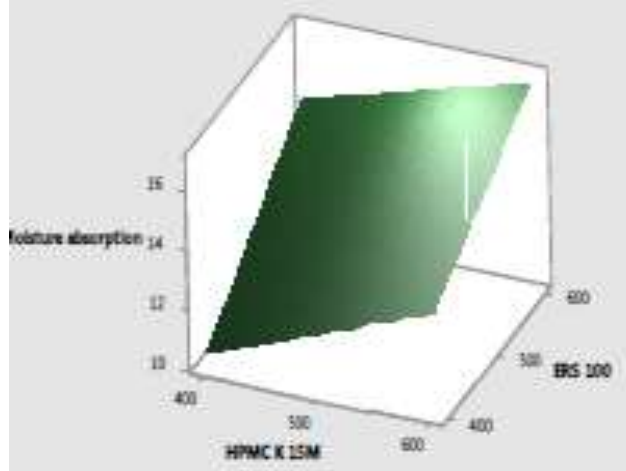

Figure 8:

Surface Plot of Cunulative \% drug release vs ERS 100, HPMCK $15 \mathrm{M}$

Cumulative\% ding release

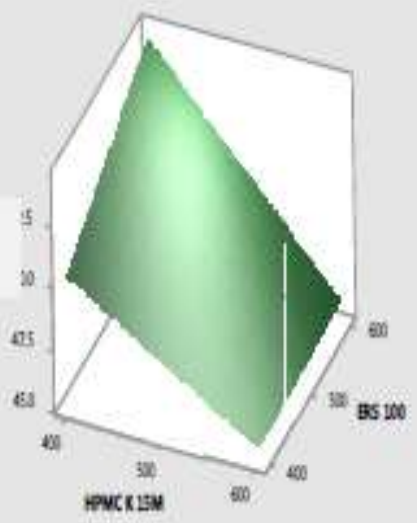

Figure 10: 


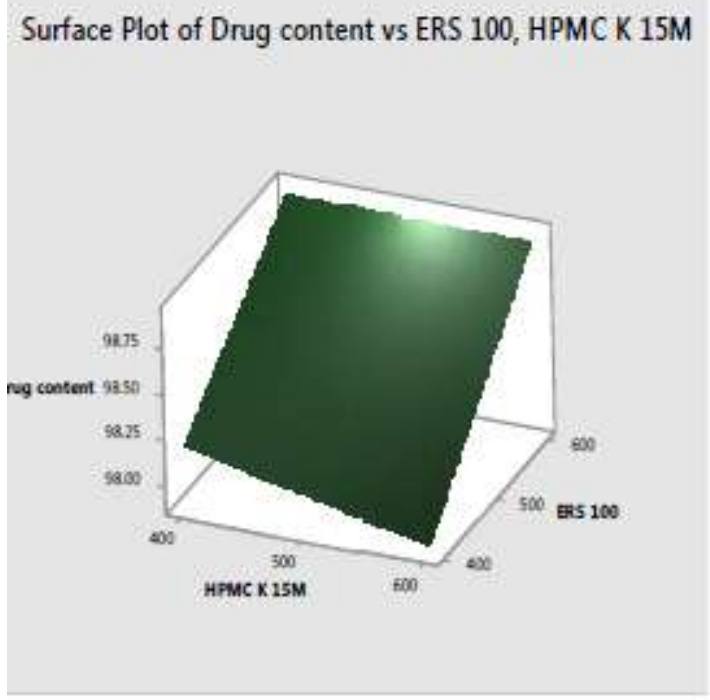

Figure 11:

Surface Plot of Moisture loss vs ERS 100, HPMC K15M

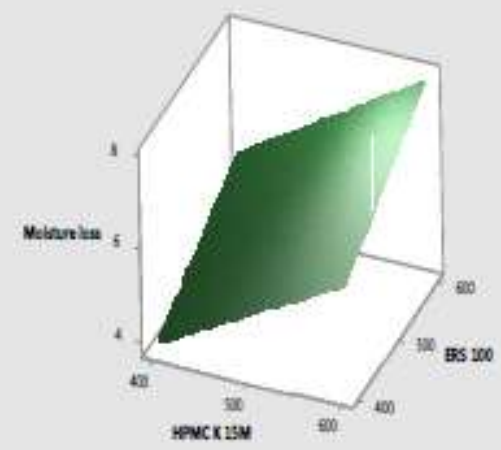

Figure 13:

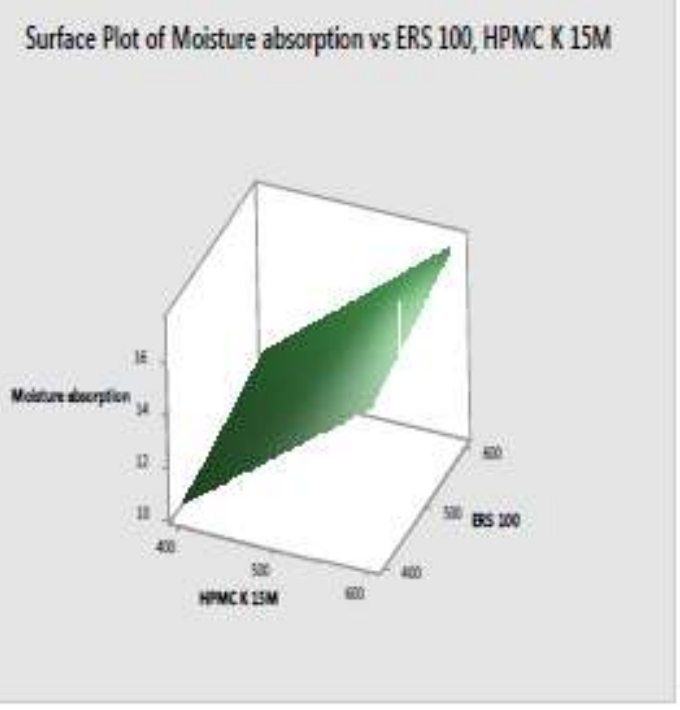

Figure 12:

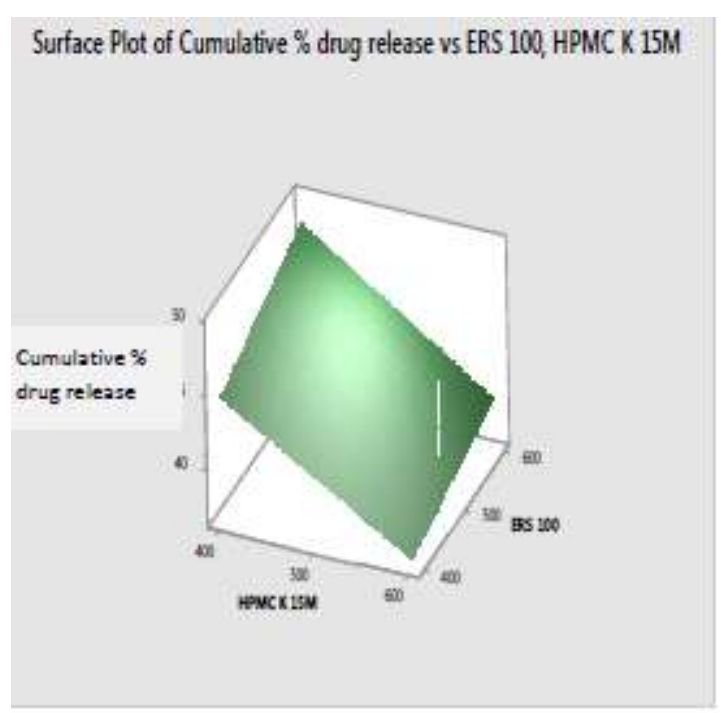

Figure 14:

\section{Stability studies}

Transdermal patches of optimized formulations (A1 \& A2) were found to be physically and chemically stable and showed no significant change in terms of physical characteristics, drug content and percentage drug release. It is evident from the stability study that the films are stable under normal shelf conditions. There were no significant physical and chemical changes in the optimized batch after 2 months. The elasticity of patches was found to be maintained (Table 2). 
Table 2: \% drug content \& \% cumulative drug release during stability study

\begin{tabular}{lllll}
\hline \multirow{2}{*}{ Time in days } & \multicolumn{2}{l}{ \% drug content } & \multicolumn{2}{l}{ \% cumulative drug release at 12h } \\
\cline { 2 - 5 } & $\mathbf{A 1}$ & $\mathbf{A 2}$ & $\mathbf{A 1}$ & $\mathbf{A 2}$ \\
\hline 0 & 99.85 & 98.92 & 54.26 & 48.3 \\
30 & 99.79 & 98.80 & 54.20 & 48 \\
60 & 99 & 99.30 & 53 & 47.6 \\
\hline
\end{tabular}

\section{In-vitro drug release kinetic study}

It was observed that the best formulation i.e. that of Almotriptan Malate loaded HPMC K 15MERS 100 for low (A1), as well as high dose(A2), followed the zero order kinetics. The correlation coefficient $(\mathrm{R})$ values were higher in the zero order model when compared to other models. So, it was concluded that this formulation follows zero order kinetics, which releases the drug in control manner and it is the ideal method of drug release to achieve pharmacological prolonged action (Table 3)

Table 3: In-vitro drug release kinetic study

\begin{tabular}{llll}
\hline No & Model & \multicolumn{2}{c}{$\mathbf{R}^{\mathbf{2}}$} \\
\cline { 3 - 4 } & & $\mathbf{A 1}$ & $\mathbf{A 2}$ \\
\hline 1 & Zero order & 0.9462 & 0.9674 \\
2 & First order & 0.5855 & 0.6592 \\
3 & Higuchi & 0.6265 & 0.6137 \\
4 & Korsmeyer \& Peppas & 0.9250 & 0.9457 \\
\hline
\end{tabular}

\section{CONCLUSION}

From the present investigation, it may be concluded that such matrix type transdermal patches of Almotriptan Malate may provide sustained transdermal delivery for prolonged periods in the management of migraine, which can be the best way to bypass the extensive hepatic first-pass metabolism. The result of the study showed the feasibility of formulating rate-controlled transdermal films of Almotriptan Malate for effective control and prophylaxis of migraine. Further in-vivo investigations are required to correlate in-vitro permeation studies for the development of a suitable transdermal system of Almotriptan Malate.

\section{ACKNOWLEDGMENTS}

The authors sincerely thank Dr. M. Gopal Rao, Professor \& Head, Department of Pharmaceutics, College of Pharmacy, Sri Ramakrishna Hospital Campus, Coimbatore for providing necessary facilities to carry out this work.

\section{REFERENCES}

1. Aggarwal.M., Puri, V., Puri S. Serotonin and CGRP in migraine. Annals of Neurosciences 2012; 19: 88-94. 
2. Volans GN. Migraine and drug absorption. Clin Pharmacokinet. 1978; 3: 313-318.

3. Per Gisle Djupesland. Nasal drug delivery devices: characteristics and performance in a clinical perspective-a review Drug Deliv Transl Res. 2013 Feb: 42-62.

4. Eliane Fuseau, Olivier Petricoul, Katy H P Moore. Clinical Pharmacokinetics of Intranasal Sumatriptan Clin. Pharmacokinet. 2002; 41: 801-11.

5. Nirav S Sheth, Rajan B Mistry. Formulation and evaluation of transdermal patches and to study permeation enhancement effect of eugenol, J Appl Pharm Sci 2011; 01: 96-101】

6. 6. Suneetha Cherukuri, Uma Maheshwari Batchu, Mandava Kiranmai, Vidhyullatha Cherukuri, KoteswaraRao Ganapuram,Formulation and evaluation of transdermal drug delivery of topiramate, Int J Pharm Investig, 2017; 7: 10-17.

7. Prabhakar D, Sreekanth J, Jayaveera KN, Development and evaluation of transdermal patches of azelnidipine. Int J Pharm Pharm Sci. 2013; 5: 805-10.

8. Keleb E, Sharma R, Mosa E.B, Zaljahwi A.Z.Transdermal drug delivery system-design and evaluation, International Journal of Advances in Pharmaceutical Sciences, 2010; 1: 201-211.

9. Kunal N Patel, Hetal K Patel, Vishnu A Patel, Formulation and Characterization of drug in adhesive transdermal patches of Diclofenac acid, Int J Pharm Pharm Sci.2012; 4: 296299.

10. Patel RP, Patel G, Baria A, Formulation and evaluation of transdermal patch of aceclofenac. Int J Drug Deliv 2009; 1:41-51.

11. Bharkatiya M, Nema RK, Bhatnaagar M. Designing and characterization of drug free patches for transdermal application. Int. j.pharm.sci.drug.res. 2010; 2: 35-39.

12. Patil MD, Mahapatra DK, Dangre PV. Formulation and invitro evaluation of once-daily sustained release matrix tablet of nifedipine using rate retardant polymers. Inventi Impact Pharm Tech. 2016; 4:190-196.

13. Amit Kumar Singh, Richa Pandey, Kuldeep Singh. Formulation and evaluation of antibiotic transdermal patch prepared by solvent evaporation method, BMR Medicine 2015; 2: 1-10.

14. Gajanan Darwhekar, Dinesh Kumar Jain, Vinod Kumar Patidar Asian. J. pharm. life sci. $2011 ; 1: 269-278$.

15. Sanjoy Kumar Dey, Pintu Kumar De, Tamaghna Sen, Ved Shankar Formulation and in vitro evaluation of transdermal matrix patches of diclofenac sodium Journal of Pharmacy Research 2011; 4: 3593-3596. 
16. Shailesh T, Prajapati, Charmi G Patel, Chhgan N Patel Formulation and evaluation of transdermal patch of Repaglinide, ISRN Pharmaceutics 2011; Article ID 651909, 9 Pages.

17. V.A. Deore, RS Kumar, PS. Gide Development and Statistical Optimization of Mucoadhesive Buccal patches of Indomethacin: In-vitro and Ex-vivo evaluation, International Journal of Advances in Pharmacy, Biology and Chemistry 2013; 2: 405422.

18. Latheeshjlal L, Phanitejaswini P, Soujanya Y, Swapna U, Sarika V, Moulika G. Transdermal Drug Delivery Systems: An Overview, Int. J. Pharmtech Res. 2011; 3: 2140-2148.

19. Andre Luis Morais Ruela, Eduardo Costa Figueirdo, Aline Gravinez Perissinato. Invitro evaluation of transdermal nicotine delivery systems commercially available in Brazil Braz. J. Pharm. Sci 2013; 49: 578-588.

20. Lincy John, Arun Kumar, Sandra Samuel. Formulation and evaluation of Amlodipine transdermal patches using ethyl cellulose. Int Res J Pharm 2013; 4: 84-88. 